\title{
LA-UR -90-3255
}

LA-UR- $-90-3255$

DE91 000214

\section{TITLE: CROSS SECTION AND ANALYZING POWER FOR QUASIFREE $(p, n)$ REACTIONS}

Author(s): T. N. Taddelicci, R. C. Byrd, T. A. Carey, J. B. McClelland, L. J. Rybarcyk, W. C. Sailor, Los Alamos National Laboratory; D. E. Ciskowski, University of Texas, Austin; .C. D. Goodman, W. Huang, Indiana University; E. Gulmez, University of CaliforniaLos Angeles; D. Marchlenski, E. Sugarbaker, Ohio State University; D. Prout, University of Colorado-Boulder; and J. Rapaport, Ohio University

$$
\begin{aligned}
& \text { Bown in } \\
& \text { OCTO } 1990
\end{aligned}
$$

Submitted to: The Proceedings of the PANIC XII, International Conference and Particles and Nuclei. Cambridge, MA, June 25-29, 1990 
T.N. Taddeucci, (a) R.C. Byrd, (a) T.A. Carey, (a) D.E. Ciskowski, (g) C.D. Goodman, (b) E. Gülmez, (e) W. Huang, (b) D. Marchlenski, (d) J.B. McClelland, (a) D. Prout, (f) J. Rapaport, (c) L.J. Rybarcyk, (a) W.C. Sailor, (a) and E. Sugarbaker, ${ }^{(d)}$

(a) Los Alamos National Laboratory, Los Alamos, NM 87545

(b) Indiana University, Bloomington, IN 47405, USA

(c) Ohio University, Athens, OH 45701, USA

(d) Ohio State University, Columbus, OH 43210, USA

(e) University of California, Los Angeles, CA 90024, USA

(f) University of Colorado, Boulder, C0 80309, USA

(g) University of Texas, Austin, TX 78712, USA

Cross sections and analyzing powers have been measured for ${ }_{12} \mathrm{C}(\mathrm{p}, \mathrm{n})$ at $494 \mathrm{MeV}$ and $795 \mathrm{MeV}$ and for $\mathrm{Pb}(\mathrm{p}, \mathrm{n})$ at $795 \mathrm{MeV}$. The data span an energy loss range of at least $200 \mathrm{MeV}$, which is sufficient to clearly reveal the large peak associated with quasifree neutron knockout. The kinematics, cross section, and analyzing power for this peak are compared to the corresponding observables for free nucleon-nucleon scattering and to the observables calculated with a relativistic Fermi-gas model.

Relativistic models of nucleon-nucleus scattering have enjoyed great success in describing elastic scattering observables. A central feature of such models is a description of the free nucleon-nucleon (NN) amplitudes in terms of four-component Dirac spinors. The lower-components of these spinors are enhanced in the nuclear medium, and this enhancenent leads to changes in the nucleon-nucleus observables. ${ }^{1,2}$ Quasifree scattering (QFS), in which the projectile makes a single hard collision with a bound target nucleon and ejects it into the continuum, provides important complementary information about the NN interaction for inelastic reaction channels.

The isovector response probed by quasifree $(p, n)$ reactions is of particular interest because of its relationship to the strength and momentum-transfer dependence of the residual particle-hole interaction. 3,4 Collectivity induced by this interaction is expected to produce significant differences between the isovector spin longitudinal $(\vec{\sigma} \cdot \vec{q})$ and spin transverse $(\vec{a} \times \vec{q})$ responses at a momentum transfer of about $1.75 \mathrm{fm}^{-1}$. In principle, the two responses can be experimentally distinguished by measuring complete sets 
of polarization transfer observables. ${ }^{5,6}$ However, the observables depend upon both the nuclear response and the effective NN interaction. A thornigh understanding of how the NN interaction changes in the nuclear medium is therefore necessary in order to extract information about the nuclear response alone.

Relativistic calculations for $\left(p, p^{\prime}\right)$ quasifree scattering have, met with mixed success.1,2,7-9 Experimental values for the analyzing power (or polarization) are significantly reduced from the free $N N$ values, and this reduction arises in a natural way in the relativistic description. Other spin observables are not as well reproduced, either in magnitude or energy-loss dependence.

In this contribution we present cross section spectra for quasifree $(p, n)$ reactions on ${ }^{12} \mathrm{C}$ and $\mathrm{Pb}$ for $\mathrm{E}_{\mathrm{p}}=795 \mathrm{MeV}$, and analyzing power data for ${ }^{2} \mathrm{C}(\mathrm{p}, \mathrm{n}) \quad$ QFS at $494 \mathrm{MeV}$ and $795 \mathrm{MeV}$. The data were obtained with the neutron time-of-flight (NTOF) facility ${ }^{10}$ at the Clinton $P$. Anderson Meson Physics Facility (LAMPF) in Los Alamos. The NTOF detector system consists of three liquid scintillator tanks and has been described elsewhere. 10 The detector system was designed as a neutron polarimeter and can be operated in a double-scattering derection mode that allows kinematic selection of $(n, n)$ and $(n, p)$ events in the scintillator. When operated in this mode a high degree of background rejection is obtained.

Spectra of cross section (lab frame) versus projectile energy loss $\omega=$ $E_{p}-E_{n}$ are presented in FIg. 1. One of the most striking features of the data shown here is the shift in the peak of the quasifree distribution relative to the energy loss expected for free NN scattering. The free NN location is marked for each spectrum by a dashed vertical line. The energy shift is approximately $26 \mathrm{MeV}$ toward higher energy loss and is nearly independent of scattering angle and bombarding energy. This shift is a common feature of the $(p, n)$ spectra for all nuclides studied.

Quasifree cross sections calculated with the relativistic Fermi-gas model of Horowitz and Murdock ${ }^{2}$ are compared to the data in Fig. 1. The dashed curves correspond to calculations employing free nucleon spinors. The calculated distributions have been shifted by a constant value of $+26 \mathrm{MeV}$ and have been normalized with effective neutron numbers two times larger than those calculated by Horowitz and Murdock. These simple calculations do a very good job of describing the main features of the data. Models that more accurately account for the surface-peaked nature of this reaction, such as the semi-infinite slab (SIS) model of Bertsch, Esbensen, and Scholten, 11-13 produce long tails on the calculated distributions and would result in an improved description of the data for higher energy losses. 

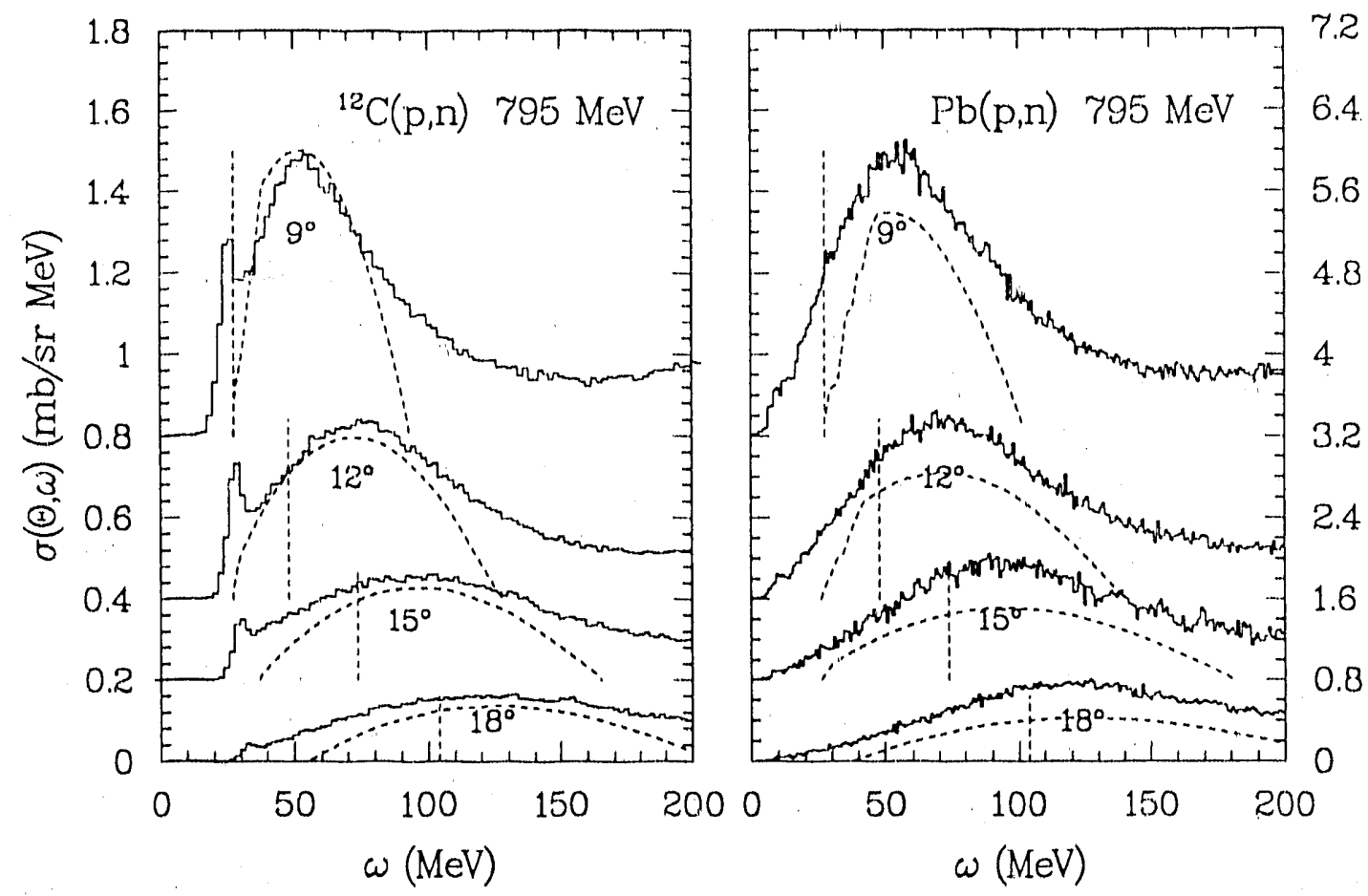

Fig. 1

Cross section spectra (lab frame) for $12 \mathrm{C}(\mathrm{p}, \mathrm{n})$ and $\mathrm{Pb}(\mathrm{p}, \mathrm{n})$ at $795 \mathrm{MeV}$. The energy loss for free scattering is indicated by vertical dashed 1ines. The curved dashed lines represent Fermi-gas calculations discussed in the text.

Analyzing-power angular distributions for the ${ }^{12} \mathrm{C}(\mathrm{p}, \mathrm{n})$ quasifree peak are plotted in Fig. 2. In relativistic models of $\left(p, p^{\prime}\right)$ reactions this observable is quite sensitive to cancellations between the large isoscalar Lorentz scalar and vector potentials. ${ }^{1,2}$ The isovector $N N$ interaction is dominated by the $\pi N$ term, however, and the analyzing power is therefore not controlled by delicate cancellations between large terms. For charge exchange reactions, deviations of the QFS observables from the free $N N$ values iesult mainly from the off-shell extrapolations of the NN interaction. Observables such as the analyzing power should therefore at some level test whether the $\pi \mathrm{N}$ coupling is best represented by a pseudoscalar or a pseudovector form. ${ }^{2}$

The experimental quasifree $(p, n)$ analyzing powers show a slight enhancement with respect to the free values at $494 \mathrm{MeV}$. The relativistic calculations with a pseudoscalar interaction seem to reproduce the magnitude and angular dependence of the data quite well. At $795 \mathrm{MeV}$, however, the quasifree analyzing powers are smaller than the free values and the pseudovector interaction gives a good description of the data. The relativistic calculations shown here are based on SM86 phase shifts of Arndt and Roper. ${ }^{14}$ A later phase shift solution (SM88, long solid line) produces a larger $795-\mathrm{MeV}$ analyzing power, however. A relativistic calculation 

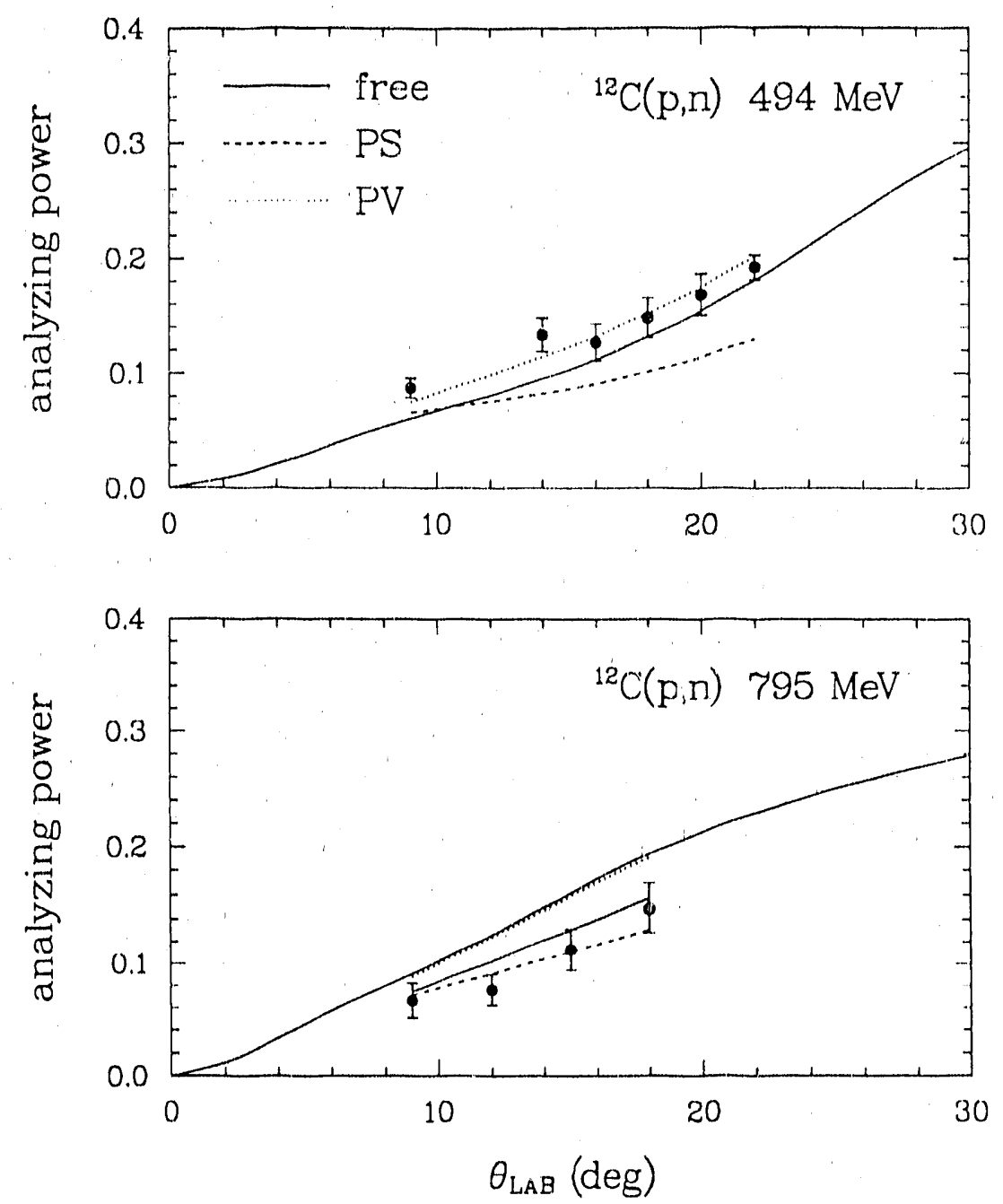

Fig. 2

Analyzing power for ${ }^{12} \mathrm{C}(\mathrm{p}, \mathrm{n})$ quasifree scattering at $494 \mathrm{MeV}$ and $795 \mathrm{MeV}$. The data points correspond to a $20-\mathrm{MeV}$ interval centered on the QFS peak location. The long solid lines correspond to free scattering and are based on the SM88 phase shifts. The short lines that span the same region as the data points correspond to relativistic Fermi-gas calculations for free spinors (solid), pseudoscalar $\pi N$ coupling (PS) with relativistic effective masses (dashed), and pseudovector $\pi N$ coupling (PV) with relativistic effective masses (dotted).

incorporating the later phase shifts would therefore produce analyzing powers that are larger than the data.

The results presented here can be summarized as follows. Relative to free NN scattering, the QFS peak for ${ }^{12} \mathrm{C}(\mathrm{p}, \mathrm{n})$ and $\mathrm{Pb}(\mathrm{p}, \mathrm{n})$ is shifted by approximately $26 \mathrm{MeV}$ toward higher energy loss. This shift is very nearly independent of bombarding energy and scattering angle. The angular dependence of the QFS cross section is well reproduced by a relativistic Fermi gas calculation, although this simple plane-waves model does not reproduce the magnitude. The angular dependence of the QFS analyzing power tracks very well with the free NN analyzing power. At $494 \mathrm{MeV}$ the magnitude of the QFS 
analyzing power is slightly enhanced relative to free NN scattering. At 795 $\mathrm{MeV}$, the DFS analyzing powers are smaller than the free NN values.

Analyzing power is the easlest spin observable to measure, but for $(p, n)$ reactions it is not the most sensitive to relativistic effects. other observables, such as the polarization transfer coefficients $D_{N N}$ and $D_{S S}$, are expected to provide more definite signatures of medium modifications of the NN interaction. ${ }^{2}$ Measurements of a complete set of polarization transfer. observables for QFS on $\mathrm{C}, \mathrm{Ca}$, or $\mathrm{Pb}$ are currently planned for the NTOF facility at LAMPF and should be completed by the end of the 1990 running period.

An important first step in the polarization transfer measurements is the calibration of the NTOF polarimeter, 1.e. a measurement of its effective analyzing power. This has been done by measuring the asymmetry produced by neutrons from the ${ }^{14} \mathrm{C}(\mathrm{p}, \mathrm{n}){ }^{14} \mathrm{~N}(2.31-\mathrm{MeV})$ reaction at a scattering angle of zero degrees. This $\mathrm{O}^{+} \rightarrow \mathrm{O}^{+}$reaction produces neutrons with the same polarization as the incident proton beam. This calibration technique has been previously employed at lower energies. ${ }^{15}$ A representative spectrum from the LAMPF ${ }^{14} \mathrm{C}(\mathrm{p}, \mathrm{n})$ calibration data is displayed in Fig. 3. The resolution obtained in this measurement was approximately $590 \mathrm{keV}$.

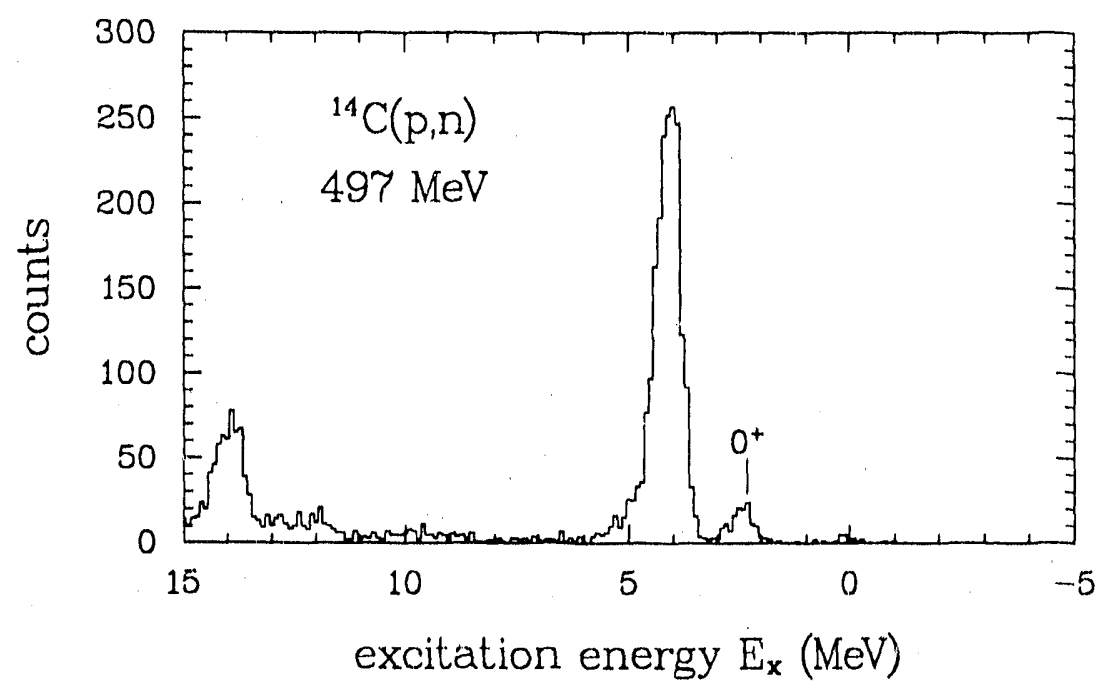

Fig. 3

Energy spectrum for ${ }^{14} \mathrm{C}(\mathrm{p}, \mathrm{n})$ at $497 \mathrm{MeV}$. The energy resolution in this spectrum (FWHM) is approximately $590 \mathrm{keV}$. Polarized neutrons from the transition to the $0^{+} 2.31-\mathrm{MeV}$ state in ${ }^{14} \mathrm{~N}$ are used to calibrate the neutron polarimeter used at the NTOF facility at LAMPF. 


\section{REFERENCES}

1) C.J. Horowitz and M.J. Iqbal, Phys. Rev. C 33 (1986) 2059.

2) C.J. Horowt tz and D.P. Murdock, Phys. Rev, C 37 (1988) 2032.

3) W.M. Alberico, A. De Pace, M. Ericson, Mikkel B. Johnson, and A. Mollnari, Phys. Rev. C 38 (1988) 109.

4) M. Ichimura, K. Kawahigashi, T.S. Jorgensen, and C. Gaarde, Phys. Rev. C 39 (1989) 1446.

5) T.A. Carey, K.W. Jones, J.B. McClelland, J.M. Moss, L.B. Rees, N. Tanaka, and A.D. Bacher, Phys. Rev. Let t. 53 (1984) 144.

6) L.B. Rees, J.M. Moss, T.A. Carey, K.W. Jones, J.B. McClelland, N. Tanaka, A.D. Bacher, and H. Esbensen, Phys. Rev. C 34 (1986) 627.

7) K.Y. Chen, L.W. Swenson, F. Farzanpay, D.K. McDaniels, Z. Tang, Z. Xu, D.M. Drake, I. Bergquist, A. Brockstedt, F.E. Bertrand, D.J. Horen, J. Lisantt1, K. Hicks, M. Vetterli, and M.J. Iqbal, Phys. Lett. B205 (1988) 436 .

8) 0. Häusser, R. Abegg, R.G. Jeppesen, R. Sawafta, A. Celler, A. Green, R.L. Helmer, R. Henderson, K. Hicks, K.P. Jackson, J. Mildenberger, C.A. Miller, M.C. Vetterli, S. Yein, M.J. Iqbal, and R.D. Smith, Phys. Rev. Lett. 61. (1988) 822 .

9) R. Fergerson, J. McG111, C. Glashausser, K. Jones, S. Nanda, Sun Zuxun, M. Barlett, G. Hoffmann, J. Marshall, and J. McClelland, Phys. Rev. C 38 (1988) 2193.

10) J.B. McClelland, Can. J. Phys. 65 (1987) 633; in: Spin Observables of Nuclear Probes, edited by Charles J. Horowitz, Charles D. Goodman, and George E. Walker, Proceedings of the Telluride International Conference on Spin Observables of Nuclear Probes, March 14-17, 1988, Telluride, Colorado (Plenum, New York, 1988) pp. 183-193.

11) H. Esbensen and G.F. Bertsch, Ann. Phys. 157 (1984) 255.

12) H. Esbensen and G.F. Bertsch, Phys. Rev. C 32 (1985) 553.

13) G.F. Bertsch and 0. Scholten, Phys. Rev. C 25 (1982) 804.

14) R.A. Arndt and L.D. Roper, Scattering Analyses Interactive Dial-in (SAID) program, Virginia Polytechnic Institute and State University (unpublished).

15) T.N. Taddeuccl, C.D. Goodman, R.C. Byrd, T.A. Carey, D.J. Horen, J. Rapaport, and E. Sugarbaker, Nucl. Instrum. Methods A241 (1985) 448. 

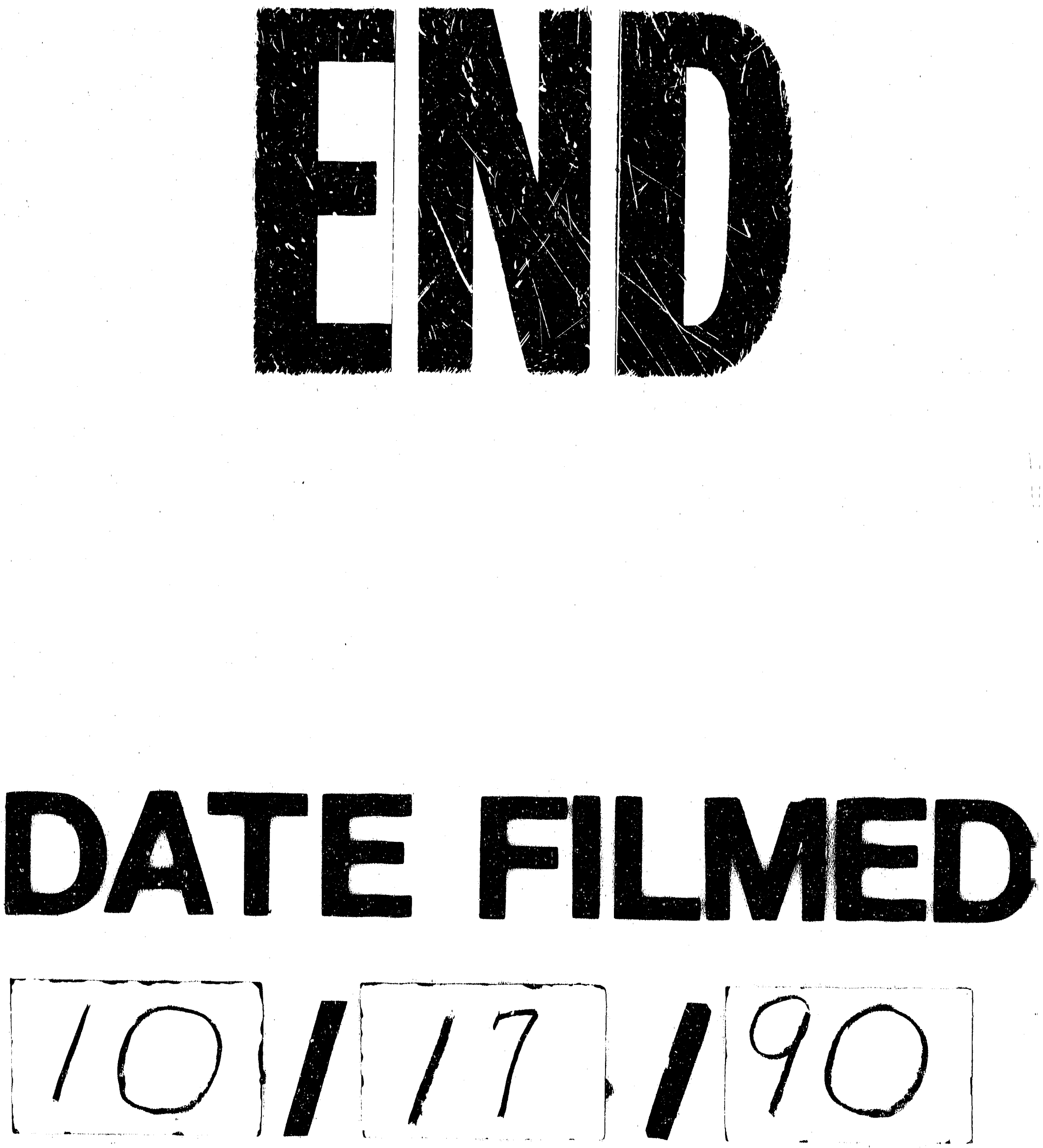
